\title{
A Case Series of Pulmonary Mucormycosis Caused by Rhizopus Microsporus
}

Mei $Y^{2 \#}$, Sun $P^{3 *}$, Wang $Z^{4}$ and Huang $X^{1 *}$

'Department of Clinical Laboratory, Children's Hospital of Nanjing Medical University, Nanjing, China ${ }^{2}$ Department of Laboratory Medicine, The First Affiliated Hospital of Nanjing Medical University, Nanjing, China ${ }^{3}$ Department of Clinical Laboratory, Northern jiangsu people's hospital, Yangzhou, China

${ }^{4}$ Liver Cancer Center, Key Laboratory of Cancer Prevention and Therapy, Tianjin Medical University Cancer Institute and Hospital, Tianjin, China "Both Authors Contributed Equally to this Work

*Corresponding author: Xu Huang, Department of Clinical Laboratory, The Affiliated Children's Hospital of Nanjing Medical University, Nanjing, China

Received: July 28, 2021; Accepted: August 20, 2021; Published: August 27, 2021

\begin{abstract}
Mucormycosis is a relatively uncommon but intractable fungal infectious disease. The mortality is very high when it occurs. In this study, we reported a continuous cases of pulmonary mucormycosis in three patients who suffered from chronic kidney disease, history of renal transplantation and multiple myeloma in April 2017, respectively. Cultures were obtained from several specimens (pathological tissue, bronchoalveolar lavage fluid and sputum) and all identified as Rhizopus microsporus by Matrix-Assisted Laser Desorption Ionization TimeOf-Flight (MALDI-TOF) mass spectrometry and ITS DNA sequencing methods. In addition, they all showed susceptibility to amphotericin B and posaconazole. Unfortunately, even through the three patients all treated with amphotericin B and posaconazole, one man still died eventually. Clinicians should keep an eye on patients who are at high risk of acquiring this fatal disease and make early intervention strategies to reduce terrible outcomes.
\end{abstract}

Keywords: Rhizopus microspores; Mucormycosis; Mass spectrometry; Microbiological

\section{Abbreviations}

MALDI-TOF: Matrix-Assisted Laser Desorption Ionization Time-Of-Flight; MALDI-TOF MS: Matrix-Assisted Laser Desorption Ionization Time-Of-Flight Mass Spectrometry; MIC: Minimum Inhibitory Concentration; SOTs: Solid Organ Transplants; HSCTs: Hematopoietic Stem Cell Transplants; HM: Hematological Malignancies; CT: Computed Tomography; SDA: Sabouraud's Agar

\section{Background}

Zygomycetes are classified into 2 orders, Mucorales and Entomophthorales [1]. Mucorales species cause mucormycosis, which is associated with high morbidity and mortality, particularly among immunosuppressed patients who are recipients of Solid Organ Transplants (SOTs) or of Hematopoietic Stem Cell Transplants (HSCTs) or who have Hematological Malignancies (HM). The oneyear cumulative TRANSNET study found that the incidence of mucormycosis was 10 -fold lower than that of aspergillosis, occurring in $0.29 \%$ of HSCTs and $0.07 \%$ of SOTs $[2,3]$. The most frequently isolated fungi causing mucormycosis were from the genus Rhizopus (47\%), in particular, Rhizopus microsporus followed by Rhizopus oryzae [4]. Mucormycosis was influenced by seasonal variations and usually occurred during the autumn months (August to November) $[4,5]$. We report a case series of disseminated mucormycosis with pulmonary infections occurred during the spring months (March to May) at the same university hospital in China.

\section{Case Presentation}

\section{Case 1}

A 52-year-old man was admitted to the hospital with clinical symptoms including a 4-year history of urine with foam and a history of pain and swelling in the lower limbs for 3 less than weeks. After a diagnosis of chronic kidney disease was made, the patient self- administered corticosteroids for a long time and underwent surgery for an arteriovenous fistula to prepare for multiple dialysis treatments. He underwent a CT-guided percutaneous lung biopsy three days after admission due to a continuous cough, the biopsy findings revealed nonseptate hyphae with acute and chronic inflammation (Figure $2 \mathrm{~A}$ ), and the tissue samples were positive on culture for Rhizopus microsporus, which was identified by Matrix-Assisted Laser Desorption Ionization Time-Of-Flight Mass Spectrometry (MALDITOF MS). Tragically, these results were obtained too late, and the patient did not receive antifungal treatment before he was discharged. In addition, a repeat chest Computed Tomography (CT) scan that was performed when he was discharged showed shrinkage of the lesion in the left upper lung lobe, while a chest CT scan performed at that time showed a lesion with cavitations in the left upper lung lobe and a lesion in the right upper lung lobe with a ground-glass appearance (Figure 1A). Finally, he discharged himself from the hospital with consent from the clinicians.

\section{Case 2}

A 44-year-old male patient underwent renal transplantation for rapidly progressive renal failure. He was on multiple maintenance immunosuppressive agents (tacrolimus, basiliximab, mycophenolate mofetil, antithymocyte globulin and methylprednisolone) and prophylactic antibiotics (acyclovir, cotrimoxazole and posaconazole) due to delayed graft function and an elevated serum creatinine after renal transplantation. Four weeks later, he presented with complaints of chest distress and a cough with sputum. At that time, his CRP level $(46.36 \mathrm{mg} / \mathrm{L})$ was markedly elevated. A chest CT scan revealed a diffuse ground glass-like lesion in the left upper lung lobe (Figure 1B). Due to an increasingly severe cough, bronchoscopy with bronchoalveolar lavage and transbronchial biopsy were performed and revealed extensive tissue necrosis with large irregular and nonseptate hyphae observed under a light microscope (Figure 2B). In 

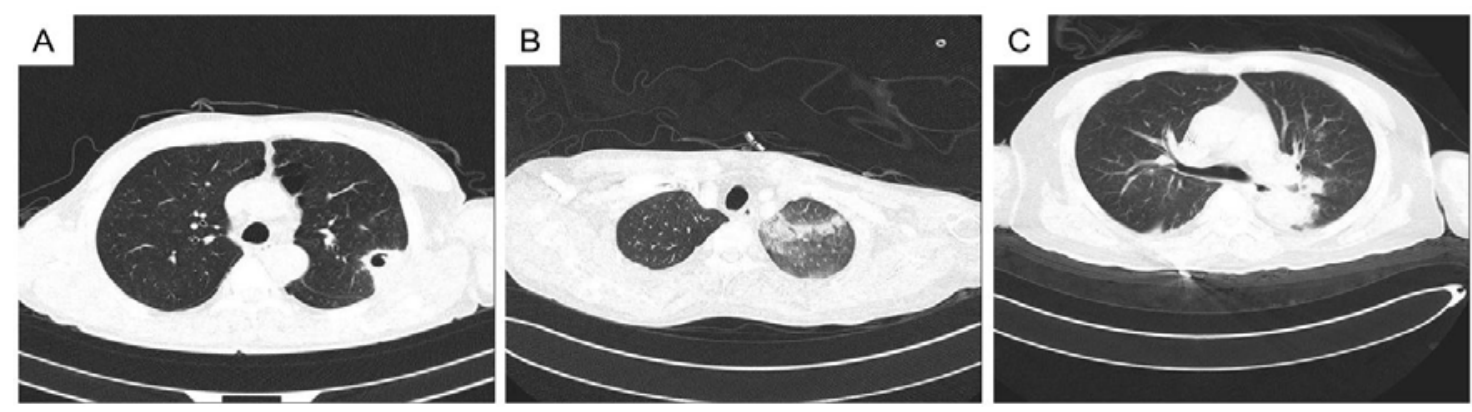

Figure 1: Chest CT scan of pulmonary mucormycosis. (A) Case 1. A left upper lung lobe lesion with cavitations and a right upper lung lobe lesion with a groundglass appearance. (B) Case 2. A left upper lobe lung lesion with diffuse bilateral ground-glass opacification. (C) Case 3 . Two upper and right lower lobe lung lesions with multiple patchy, nodular and high-density shadows.
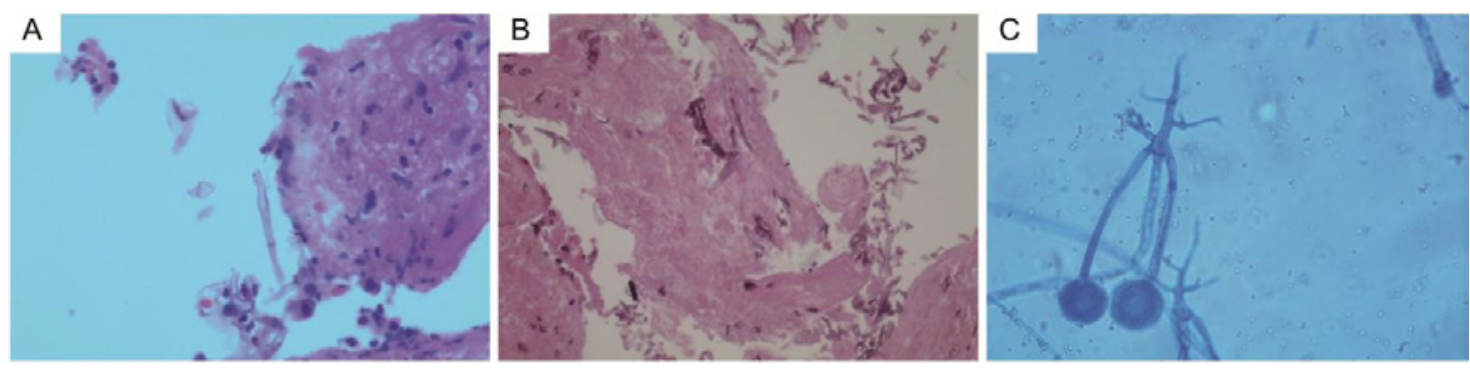

Figure 2: Histopathological examination of lung tissue by periodic acid-Schiff staining (PAS). (A) Case 1. Nonseptate hyphae with acute and chronic inflammation, 400x. (B) Case 2. Extensive tissue necrosis and large irregular nonseptate hyphae, 400x. (C) Microscopic view of the isolate on Sabourauds agar showing broadbased nonseptate hyphae with dichotomous wide-angle branching, 400x.

addition, the bronchoalveolar lavage sample was cultured and grew Rhizopus microsporus, which was identified by MALDI-TOF MS. In light of disseminated mucormycosis and no significant clinical improvement, a high dosage of liposomal amphotericin B (10 mg/ $\mathrm{kg} / \mathrm{d}$ IV) was administered, and his doses of immunosuppressive medications were reduced. Unfortunately, he coughed a massive amount of blood 8 weeks after admission and died.

\section{Case 3}

A 56-year-old man who presented with sacrolumbar pain was admitted to hospital. He was diagnosed with multiple myeloma and underwent chemotherapy with cyclophosphamide, velcade and thalidomide in the hospital. He was provided antibiotic prophylaxis with cefoperazone/tazobactam and voriconazole. At first, he had a slight cough with no mucus, but his symptoms progressed, and he coughed purulent sputum on the tenth day after chemotherapy. A chest CT scan revealed two lesions in the right upper and lower lung lobes with multiple patchy, nodular and high-density shadows (Figure 1C). Three consecutive cultures of the sputum grew Rhizopus microsporus, which was identified by MALDI-TOF MS. A high dosage of liposomal amphotericin B (10 mg/kg/d IV) was administered, and voriconazole was used instead of posaconazole ( $400 \mathrm{mg}$ twice daily, taken orally) as soon as the patient was diagnosed with mucormycosis. The patient stopped amphotericin B when he developed gastrointestinal hemorrhage. After treatment with posaconazole for approximately 4 months, the infection was controlled, which was further confirmed by a negative sputum culture, and the patient's symptoms disappeared.

\section{Discussion and Conclusions}

Mucormycosis is an emerging angioinvasive infection caused by ubiquitous filamentous fungi of the order Mucorales that commonly affect the sinuses (39\%) and the lungs (24\%) $[5,6]$. To establish infection, Mucorales species form spores that are small (3-11 $\mu \mathrm{m})$ enough to reach the distal alveolar spaces and then overcome phagocytosis by macrophages and neutrophils to germinate into hyphae [3]. Clinical features of our 3 patients with pulmonary infections caused by Rhizopus microsporus were summarized in Table 1. In particular, the patient who underwent renal transplantation (Case 2) asphyxiated with hemoptysis. In the past, some patients formed a mucor embolus that blocked the bronchi and resulted in lung collapse and invasion of the hilar blood vessels with subsequent massive hemoptysis $[7,8]$. The risk factors for mucormycosis in our 3 patients included immunosuppressive regimens, corticosteroid therapy, renal transplantation, dialysis treatments and chemotherapy. However, one patient (Case 1) seemed to have no obvious serious immune deficiency, and his prognosis could not be traced. As we know, Rhizopus microsporus is an opportunistic pathogen that can cause serious and often life-threatening infections.

An unprecedented outbreak of intestinal zygomycosis due to Rhizopus microsporus occurred in Hong Kong, and similar cases have been reported in the USA and elsewhere in Europe $[9,10]$. These reports suggest that timely diagnosis and treatment can not only cure existing cases but also prevent further transmission. The diagnosis of an invasive fungal infection primarily requires comprehensive lab examinations including microbiological, imaging and pathological evaluations as well as a high index of clinical suspicion. However, imaging and pathology examinations have limitations for identifying the organism at the genus and species levels. Therefore, isolation of fungi by culture is vital for definitive identification. Isolates from 
Table 1: Clinical features of three patients with pulmonary infection caused by Rhizopus microspores.

\begin{tabular}{|c|c|c|c|}
\hline Clinical feature & Case 1 & Case 2 & Case 3 \\
\hline Age in years & 52 & 44 & 56 \\
\hline Gender & Male & Male & Male \\
\hline Hospital stay & $\begin{array}{l}2017 / 3 / 28- \\
2017 / 4 / 15\end{array}$ & $2017 / 2 / 23-2017 / 4 / 20$ & $2017 / 4 / 4-2017 / 5$ \\
\hline Underlying disease & $\begin{array}{l}\text { Chronic kidney } \\
\text { disease }\end{array}$ & After renal transplanation & Multiple myeloma \\
\hline \multicolumn{4}{|l|}{ Symptoms } \\
\hline Fever & Yes & Yes & Yes \\
\hline Cough & Yes & Yes & Yes \\
\hline \multicolumn{4}{|l|}{ Diagnostic method } \\
\hline Imaging data (X ray, CT scan) & Yes & Yes & Yes \\
\hline Culture & Yes & Yes & Yes \\
\hline $\begin{array}{l}\text { beta-D-glucan, galactomannan } \\
\text { antigen }\end{array}$ & negative & negative & negative \\
\hline \multicolumn{4}{|l|}{ Therapy } \\
\hline Prophylactic antibotics & No & Acyclovir; Cotrimoxazole; Posaconazole & $\begin{array}{c}\text { Cefoperazone-azobactam } \\
\text { Voriconazole }\end{array}$ \\
\hline Antifugal therapy after culture & No & Liposomal amphotericin B & $\begin{array}{c}\text { Liposomal amphotericin B } \\
\text { Posaconazole }\end{array}$ \\
\hline Surgical intervention & No & Yes & No \\
\hline Outcome of patient & Unknown & Expired & Recovered \\
\hline
\end{tabular}

all the patients were cultured on Sabouraud's Agar (SDA) and were positive for Rhizopus microsporus after $24 \mathrm{~h}$, which was initially identified by MALDI-TOF MS using the FDA-approved Vitek MS version MS-CE CLI 2.0.0 (bioMérieux Inc., Durham, NC). In addition, all the isolates sequenced had $100 \%$ identity to Rhizopus microsporus species using the ITS1 and ITS4 primers and the NL1 and NL4 primers. Studies have explored MALDI-TOF MS as a rapid, simple and correct identification technique for the species and variety levels of 38 strains of Rhizopus [11]. Obviously, MALDI-TOF MS requires a shorter period for identification than molecular methods that require culturing, DNA extraction, PCR and sequencing. When used in combination with surgical intervention, this technique may greatly improve diagnostic efficiency and accuracy $[6,11]$. As noted in these cases, invasive lung biopsy can provide a more reliable specimen for identification. On microscopic examinations, these fungi displayed broad-based nonseptate hyphae with dichotomous wide-angle branching (Figure 2C). In addition, the chest CT scans of these 3 patients showed lung lesions with inflammation. With the exception of one patient who had a lack of pathological data (Case 3 ), the invasive lung tissue pathology of the other two patients (Case 1 and Case 2) both showed serious inflammation and nonseptate hyphae. As seen in our 3 consecutively admitted patients, doctors and laboratory personnel cooperated with each other and summarized findings to improve the diagnosis and efficiency from the initial pity. Perhaps a potential outbreak was unknowingly avoided.

Aggressive antifungal drugs combined with local surgical debridement is a known promising therapeutic combination for mucormycosis [3,12-14]. In particular, the timely initiation of a liposomal formulation of amphotericin B monotherapy is the firstline treatment for mucormycosis [3]. Posaconazole and isavuconazole are acceptable salvage and long-term treatment options when patients are at a high risk of amphotericin-induced nephrotoxicity $[15,16]$. Mucorales species are innately highly resistant to echinocandins that can be used effectively for aspergillosis, so we did not evaluate the MICs for this class of drugs for treating Rhizopus microsporus. Antifungal susceptibility testing was performed in accordance with the CLSI guidelines M38-A2 [17]. The MICs were obtained by visual inspection after incubation at $35^{\circ} \mathrm{C}$ for $24 \mathrm{~h}$ for Rhizopus spp. The results showed that all isolates were susceptible to amphotericin $\mathrm{B}$ (MIC $=0.125 \mu \mathrm{g} / \mathrm{ml}$ ) and posaconazole $(\mathrm{MIC}=0.125 \mu \mathrm{g} / \mathrm{ml})$. The MIC ranges of voriconazole and itraconazole for these 3 isolates were 1 to 8 and 16 to $32 \mu \mathrm{g} / \mathrm{ml}$, respectively. The results of antifungal susceptibility testing were similar to those in previous reports $[15,18,19]$. One patient did not receive any antifungal therapy, while the other two patients who were treated with amphotericin B and posaconazole showed treatment failure and a favorable prognosis, respectively.

Although all the isolates were susceptible to amphotericin $\mathrm{B}$ and posaconazole, the poor prognosis of one patient (Case 2) might be related to the rapid progression of underlying disease and angioinvasive infection by Rhizopus microsporus. Fortunately, one patient (Case 3) remained asymptomatic after a 4-month course of posaconazole.

In conclusion, mucormycosis is an angioinvasive and progressive infection associated with high morbidity and mortality. The diagnosis and treatment of mucormycosis is often clinically challenging in part due to difficult identification of the etiologic agent and rapid progression of disease. Timely prevention, early diagnosis and comprehensive treatment are effective ways to prevent mucormycosis from spreading and to improve patient outcomes despite the low survival rates in disseminated cases. 


\section{Authors' Contributions}

YM contributed to design the study. ZYW performed the research $\mathrm{XH}$ made critical revisions to the manuscript and contributed to concept/design. PFS made critical revisions to the manuscript. All the authors have read and approved the final revision to be published.

\section{Availability of data and materials}

Materials in the form of electronic medical records are available by inquirying the corresponding author.

\section{Ethics approval and consent to participate}

This study was approved by the Ethics Committee of the hospital and was in accordance with the ethical guidelines of Declaration of Helsinki. Each participant provided written informed consent before the study.

\section{Consent for publication}

Written informed consent was obtained from the patient's spouse, his medical power of attorney, for publication of this case report. The patient provided verbal assent.

\section{References}

1. Kwon-Chung $\mathrm{KJ}$. Taxonomy of fungi causing mucormycosis and entomophthoramycosis (zygomycosis) and nomenclature of the disease: molecular mycologic perspectives. Clin Infect Dis. 2012; 54: S8-S15.

2. Pappas PG, Alexander BD, Andes DR, et al. Invasive fungal infections among organ transplant recipients: results of the Transplant-Associated Infection Surveillance Network (TRANSNET). Clin Infect Dis. 2010; 50: 1101-1111.

3. Park BJ, Pappas PG, Wannemuehler KA, et al. Invasive non-Aspergillus mold infections in transplant recipients, United States, 2001-2006. Emerg Infect Dis. 2011; 17: 1855-1864.

4. Farmakiotis D, Kontoyiannis DP. Mucormycoses. Infect Dis Clin North Am. 2016; 30:1 43-163

5. Petrikkos G, Skiada A, Lortholary O, et al. Epidemiology and clinical manifestations of mucormycosis. Clin Infect Dis. 2012; 54: S23-S34.

6. Harrasser $N$, Banke IJ, Hauschild $M$, et al. Clinical challenge: fatal mucormycotic osteomyelitis caused by Rhizopus microsporus despite aggressive multimodal treatment. BMC Infect Dis. 2014; 14: 488.
7. Kitabayashi A, Hirokawa M, Yamaguchi A, et al. Invasive pulmonary mucormycosis with rupture of the thoracic aorta. Am J Hematol. 1998; 58: 326-329.

8. Passamonte PM, Dix JD. Nosocomial pulmonary mucormycosis with fatal massive hemoptysis. Am J Med Sci. 1985; 289: 65-67.

9. Cheng VC, Chan JF, Ngan AH, et al. Outbreak of intestinal infection due to Rhizopus microsporus. J Clin Microbiol. 2009; 47: 2834-2843.

10. Antoniadou A. Outbreaks of zygomycosis in hospitals. Clin Microbiol Infect 2009; 15: 55-59.

11. Dolatabadi S, Kolecka A, Versteeg M, et al. Differentiation of clinically relevant Mucorales Rhizopus microsporus and R. arrhizus by matrix-assisted laser desorption ionization time-of-flight mass spectrometry (MALDI-TOF MS). J Med Microbiol. 2015; 64: 694-701.

12. Spellberg B, Walsh TJ, Kontoyiannis DP, et al. Recent advances in the management of mucormycosis: from bench to bedside. Clin Infect Dis. 2009; 48: 1743-1751.

13. Bernardo RM, Gurung A, Jain D, et al. Therapeutic Challenges of Hepatic Mucormycosis in Hematologic Malignancy: A Case Report and Review of the Literature. American Journal of Case Reports. 2016; 17: 484-489.

14. Hodel L, Inci I, Schanz U, et al. Urokinase application for hemothorax in pulmonary mucormycosis. Journal of Thoracic Disease. 2018; 10: E175-E178.

15. Chowdhary A, Kathuria S, Singh PK, et al. Molecular characterization and in vitro antifungal susceptibility of 80 clinical isolates of mucormycetes in Delhi, India. Mycoses. 2014; 57: 97-107.

16. Chitasombat MN, Kontoyiannis DP. The 'cephalosporin era' of triazole therapy: isavuconazole, a welcomed newcomer for the treatment of invasive fungal infections. Expert Opin Pharmacother. 2015; 16: 1543-1558.

17. CLSI. Reference Method for Broth Dilution Antifungal Susceptibility Testing of Filamentous Fungi; Approved Standard-Second Edition. M38-A2. Clinical and Laboratory Standards Institute,Wayne, PA. 2008.

18. Espinel-Ingroff A, Chakrabarti A, Chowdhary A, et al. Multicenter evaluation of MIC distributions for epidemiologic cutoff value definition to detect amphotericin B, posaconazole, and itraconazole resistance among the most clinically relevant species of Mucorales. Antimicrob Agents Chemother. 2015; 59: 1745-1750.

19. Almyroudis NG, Sutton DA, Fothergill AW, et al. In vitro susceptibilities of 217 clinical isolates of zygomycetes to conventional and new antifungal agents. Antimicrob Agents Chemother. 2007; 51: 2587-2590. 